Each strip represents the contribution of the waves at intervals of $1 / 60$ of a cell edge. The strips are prepared for all amplitudes between $\overrightarrow{99}$ and 99 , and all frequencies (that is, number of waves in the cell edge) up to 20. When summing a set of Fourier terms we only have to select the appropriate strips, place them one beneath the other, and sum the columns.

By using cosine and sine terms separately in preliminary and final summations, full use of the symmetries possessud by these functions can be made. It is not necessary to have the strips written out for more than one quarter of the cell edge. The cosine waves corresponding to even indexes and the sine waves corresponding to odd indexes are all symmetrical about the point $\frac{1}{4}$, while the others are anti-symmetrical about this point.

The strips are stored in boxes as shown in Fig. 2. One box contains all the cosine strips and another the sine strips, and each compartment contains the strips of one frequency. The sloping sides of the boxes ensure that when a strip is removed its place is left open for its re-insertion.

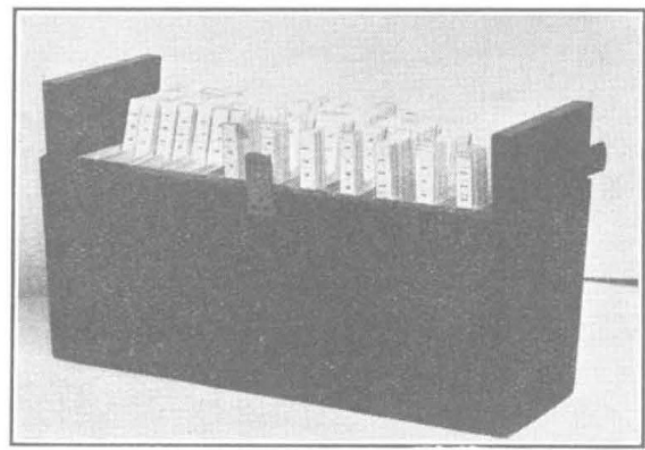

FrG. 2. Set of cosine strips in box.

The use of amplitudes from 1 to \pm 99 is adequate for all work of normal accuracy. If only a few of the totals of the preliminary tables exceed 99 , these totals can be made up by the use of two of the strips instead of one in the final tables. If a large number of the totals of the preliminary tables exceed 99 , then all these totals should be divided by some suitable factor.

It is estimated that one worker using the strips can complete a Fourier synthesis of the size of the example used here in a total time of eight hours. (A rough estimate of the length of a Fourier synthesis can be made by giving the total number of terms involved if the complete summation were done directly. In our example, $80 \mathrm{~F}$ 's are summed over 1,800 points, making 124,000 terms in all.) A further utility is that the selection and addition of the strips can be done by inexperienced persons, and the results checked with certainty by doing one line of the synthesis along one $y$-level, thus crossing all the previous $x$-levels.

We should be glad to know if workers in other laboratories who employ the Fourier method would find these sets of strips useful, as it may be possible to arrange for the supply of copies.

C. A. Beevers.

Physical Laboratories,

H. Lipson.

University, Manchester. March 7.

Z. Krist., A, 90, 517 (1935).

\section{Magnetic Anisotropy of Resorcinol}

THE diamagnetic anisotropy of a large crystal of resorcinol, weighing $0.05550 \mathrm{gm}$., has been measured by methods similar to those described by Krishnan ${ }^{1}$, with the following results:

$$
\begin{aligned}
& \chi_{a}-\chi_{c}=-5.36 \times 10^{-6} \text { o.G.s. E.M.U.; } \\
& \chi_{b}-\chi_{c}=-13.17 \times 10^{-6} \quad, \quad, \quad ; \\
& \chi_{b}-\chi_{a}=-7 \cdot 74 \times 10^{-6} \quad, \quad, \quad \text {; }
\end{aligned}
$$

$a, b, c$ being taken in accordance with Robertson's recent renaming of these axes ${ }^{2}$, whereby $a: b: c=$ $10 \cdot 53: 9 \cdot 53: 5 \cdot 66 \mathrm{~A}$. Resorcinol is very soluble in water and in all the usual organic solvents, and Rabi's method $^{3}$ for the measurement of the absolute susceptibility in a given direction could not therefore be used. The mean susceptibility of powdered resorcinol has, however, been measured by Pascal ${ }^{4}$ and using his value, $\chi_{m}=67.2 \times 10^{-6}$, we obtain $\chi_{a}=-66 \cdot 4, \chi_{b}=-74 \cdot 2, \chi_{c}=-61 \cdot 0 \times 10^{-6}$.

Now in an orthorhombic crystal

$\chi_{a}=K_{1} \cos ^{2} \alpha_{1}+K_{2} \cos ^{2} \alpha_{2}+K_{3} \cos ^{2} \alpha_{3}$ $\left.\chi_{b}=K_{1} \cos ^{2} \beta_{1}+K_{2} \cos ^{2} \beta_{2}+K_{3} \cos ^{2} \beta_{3}\right\}$. .

$\chi_{c}=K_{1} \cos ^{2} \gamma_{1}+K_{2} \cos ^{2} \gamma_{2}+K_{3} \cos ^{2} \gamma_{3}$

where $\cos \alpha_{1}, \cos \beta_{1}, \cos \gamma_{1}$, are the direction cosines of $K_{1}$ relative to $a, b, c$, and so on ; $K_{1}, K_{2}, K_{3}$ being the three principal susceptibilities of the molecule.

If $K_{1}=K_{2}$, as is approximately true for most aromatic compounds, then

$$
\begin{aligned}
& \chi_{a}=K_{1}+\left(K_{3}-K_{1}\right) \cos ^{2} \alpha_{3} \\
& \chi_{b}=K_{1}+\left(K_{3}-K_{1}\right) \cos ^{2} \beta_{3} \\
& \chi_{c}=K_{1}+\left(K_{3}-K_{1}\right) \cos ^{2} \gamma_{3}
\end{aligned}
$$

Hence if $K_{1}$ and $K_{3}$ can be estimated correctly, the direction cosines of the normal to the molecular plane can be calculated. As shown by Krishnan (loc. cit.), a good estimate of the molecular susceptibilities may be obtained by adding to each of the principal susceptibilities of benzene the difference between the mean susceptibility of benzene and that of resorcinol.

Thus $K_{1}=K_{2}=-37 \cdot 3-11 \cdot 9=-49 \cdot 2$ )

$$
\left.K_{3}=-91 \cdot 2-11 \cdot 9=-103 \cdot 2\right\}
$$

and on substituting in (2), we obtain

$$
\alpha=55 \cdot 6^{\circ}, \beta=47 \cdot 1^{\circ}, \gamma=62 \cdot 1^{\circ} \text {. }
$$

The same angles derived by Robertson from a single Fourier analysis ${ }^{5}$ are

$$
\alpha=56 \cdot 1^{\circ}, \beta=46 \cdot 9^{\circ}, \gamma=61 \cdot 3^{\circ},
$$

in good agreement with the above.

\section{Davy Faraday Laboratory,}

KathleEN Lonsdale.

$$
\text { Royal Institution, W.1. }
$$

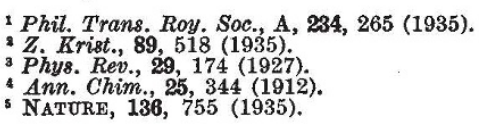

\section{Fine Structure of the $L_{23}$ Absorption Edge of Magnesium Metal}

IN the region of ordinary $\mathrm{X}$-rays, variations in the intensity of radiation transmitted through a metal foil on the short wave-length side of an absorption edge have led to interesting results in relation to the zone-structure of the unoccupied electron levels of a metal. The extension of such 'fine structure' measure. ments to absorption edges which lie in the region of ultra-soft X-rays (say 100-300 A.) allows the possibility of a considerable increase in the resolving power of the method, since in this region the breadth 